\title{
Erratum: Theory of nonretarded ballistic surface plasma waves in metal films [Phys. Rev. B 95, 125442 (2017)]
}

\author{
Hai-Yao Deng \\ (Received 15 September 2017; published 25 September 2017)
}

DOI: 10.1103/PhysRevB.96.119914

I spotted a few typographical errors in Sec. IV of the original paper which are corrected here. In the upper line of Eq. (48) and in the last line of (49), the prefactor $\frac{1}{2}$ should be removed. In the three-line expression for $\gamma_{0}$ that follows after Eq. (49), the factor $\frac{1}{2}$ appearing in the numerator of the first line should be removed, the factor $\frac{1}{4}$ appearing in the second line should be changed to $\frac{1}{2}$, and the factor $\frac{1}{2}$ in the third line should be removed. Finally, in the inline expression that follows immediately after Eq. (50), the right-hand side should be multiplied by 2 . None of the results and conclusions are affected by the typographical errors. 